\title{
Thermodynamic aspects of describing the contribution of spontaneous magnetism of electrons to the Hall resistance
}

\author{
Vsevolod Okulov ${ }^{1,2}$, and Evgeny Pamyatnykh ${ }^{2}$
}

${ }^{l}$ M.N. Miheev Institute of Metal Physics of Ural Branch of RAS 620990 Ekaterinburg, Russia

${ }^{2}$ Ural Federal University, 620083, Ekaterinburg, Russia

\begin{abstract}
On the base of the analysis of quantum-statistical description of the magnetization of electron system containing the spontaneous spin polarization contribution there were found the magnetization and conduction current densities in equilibrium state. It has been shown that equilibrium surface conduction current ensures realization of demagnetization effects but in local equilibrium state determines local equilibrium part of the Hall conductivity. As a result one is given the justification of influence of the spontaneous magnetization on galvanomagnetic effects, which is not related to spin-orbital interaction.
\end{abstract}

\section{Introduction}

Now the study of manifesting the spontaneous magnetization of electrons in the Hall effect and other galvanomagnetic phenomena is actively developed. In the present report we show that for describing the phenomena relating to anomalous Hall effect there can be used naturally the methods of equilibrium and nonequilibrium thermodynamics. The term "thermodynamic anomalous Hall effect" is introduced by us to designate anomalous Hall effect which can be described by thermodynamic methods. Next we consider the connection of thermodynamic anomalous Hall effect with equilibrium magnetization.

Necessity of such researches has been explicit after revealing in [1] the contribution to the Hall resistance of semiconductor from spontaneous magnetization of donor electrons of iron impurities of very low concentrations. Such low concentration does not admit ordinary explanation of the given effect by the manifestation of spin-orbital interaction. It is the search of other explanations that has resulted in revealing other physical mechanisms of the anomalies, which are not related to spin-orbital interaction.

\section{Equilibrium conduction current}

From the beginning of considering we choose as initial a well known formula for the density vector of equilibrium magnetization $\mathbf{M}$ of quantum system of electrons

$$
\mathbf{M}=\left(\frac{\partial \Omega}{\partial \mathbf{H}}\right)_{T \zeta}=m \mathbf{h}+\frac{1}{H} P \mathbf{h}
$$

It is obtained from thermodynamic potential $\Omega$ in variables of temperature, chemical potential $\zeta$ and magnetic field strength $\mathbf{H}$. Taking into account real inhomogeneity of a system, this formula can be transformed to the expression for varying the potential $\Omega$ with respect to vector potential of magnetic field $\mathbf{A}(\mathbf{r})$ :

$$
\begin{aligned}
& \delta \Omega=- \frac{1}{c} \int d \mathbf{r} \delta \mathbf{A}(\mathbf{r}) \mathbf{j}_{\mathrm{m}}(\mathbf{r})= \\
&=-\frac{1}{c} \int d \mathbf{r} \delta \mathbf{A}(\mathbf{r})\left[\mathbf{j}_{\mathrm{eq}}(\mathbf{r})-\mathbf{j}_{\mathrm{c}}(\mathbf{r})\right]
\end{aligned}
$$

In this equality $\mathbf{j}_{m}(\mathbf{r})=c$ rot $\mathbf{M}$ is the magnetization current density, $\mathbf{j}_{e q}(\mathbf{r})=c$ rot $\mathbf{m}$ is the density of mean magnetic moment flux of an electron and we assume that the vectors $\mathbf{H}, \mathbf{M}$ and $\mathbf{m}$ are directed along the symmetry axis. Then the vector $\mathbf{j}_{c}(\mathbf{r})$ is directed perpendicularly to $\mathbf{H}$ and defined by the equality:

$$
\frac{1}{c}\left[\mathbf{j}_{c}(\mathbf{r}) \cdot \mathbf{H}\right]=\operatorname{grad} P
$$

Here $P=-(\Omega / V)$ is the pressure of electron system. This equality is the condition of the equilibrium of the external force, with which magnetic field acts the system with current, and the pressure force of the system. It means that the quantity $\mathbf{j}_{c}(\mathbf{r})$ should be considered as the conduction current density, due to existence of the external force mentioned above. If the system has a spontaneous magnetization $M_{0}$, the pressure in (3) includes the energy $M_{0} H$. In homogeneous equilibrium system the currents considered are concentrated at the boundaries. According to the equation (3) the magnitude and distribution of equilibrium conduction current depend on the structure and shape of the boundaries and it is this current that is responsible for the existence of so-called demagnetizing field. Simple definition of such a field can be represented by Maxwell equations inside the boundaries of a system:

$$
\begin{aligned}
& \operatorname{rot} \mathbf{B}_{e q}-4 \pi \cdot \operatorname{rot} \mathbf{M}=\operatorname{rot} \mathbf{H}_{e q}=\frac{4 \pi}{c} \mathbf{j}_{c}(\mathbf{r}) \\
& \operatorname{div} \mathbf{H}_{e q}=0,
\end{aligned}
$$


( $\mathbf{B}_{e q}, \mathbf{H}_{e q}$ are the induction and strength vectors of magnetic field) and outside the boundaries of a system one has:

$$
\begin{aligned}
\operatorname{rot} \mathbf{H}_{e} & =\frac{4 \pi}{c} \mathbf{j}_{e}(\mathbf{r})+\frac{4 \pi}{c} \mathbf{j}_{c}(\mathbf{r}) \\
\operatorname{div} \mathbf{H}_{e} & =0
\end{aligned}
$$

$\left(\mathbf{H}_{e}\right.$ and $\mathbf{j}_{e}(\mathbf{r})$ are the magnetic field strength and current density of the field source). Equations (4) and (5) describe a modification of the initial magnetic field strength $\mathbf{H}$ inside the system by demagnetizing coefficients, which is specified by the current of a source $\mathbf{j}_{e}(\mathbf{r})$. As a whole the role of conduction current, determined by us, can been characterized by averaged value of $\mathbf{J}_{c}$, which is obtained by integrating the equation (4) over the layer, adjoining to the boundary, and in the plane of cross-section, perpendicular to the magnetic field strength one has:

$$
H \cdot \mathbf{J}_{c}=-\frac{c}{s} \int d \mathbf{r} \operatorname{rot}(P \mathbf{h})
$$

$s$ is the area of integrating.

\section{The Hall current}

The presented ideas about equilibrium conduction current can be applied to studying the Hall resistance, as far as it contains the contribution from the current of local equilibrium. Local equilibrium in the field of current source with potential $\varphi(\mathbf{r})$ is described by adding the energy $e \varphi(\mathbf{r})$ to chemical potential. Extending the results, quoted above, to local equilibrium state of electron system and replacing $P(\zeta)$ by $P(\zeta+e \varphi(\mathbf{r}))$ in the right-hand part of (3), one obtains additional term

$$
-e n \mathbf{E}-e \mathbf{E} \frac{\partial M_{0}}{\partial \zeta},
$$

where $\mathbf{E}=-\operatorname{grad}(\varphi(\mathbf{r}))$ is the electric field strength, $n=\partial P / \partial \zeta$ is the electron concentration.

Then averaged equilibrium conduction current, defined by equation (6), is replaced by local equilibrium one

$$
\begin{aligned}
\mathbf{J}_{c}^{L}= & \mathbf{J}_{c}+\left\{\frac{e c n}{H}+e c \frac{\partial M_{0}}{\partial \zeta}\right\}[\mathbf{E} \cdot \mathbf{h}]= \\
& =\mathbf{J}_{c}+\left(\sigma_{H}^{L}+\sigma_{m}\right)[\mathbf{E} \cdot \mathbf{h}]=\mathbf{J}_{c}+\mathbf{J}_{H}^{L}
\end{aligned}
$$

In this expression as a term of local equilibrium current there enters well known contribution of local equilibrium to the Hall current with conductivity $\sigma_{H}^{L}$, which is predominant in high magnetic field and also the contribution of spontaneous magnetization with the conductivity $\sigma_{m}$ revealed first in work [1], and the contribution of equilibrium conduction current $\mathbf{J}_{c}$ represented by us in the present work. The terms reflecting the manifestation of spontaneous magnetization of electron system in galvanomagnetic phenomena are of fundamental importance.

\section{Final remarks}

It should be noted that the Hall conductivity, considered by us, includes also the diagonal element of electrical resistivity tensor in magnetic field. Thus in the given work we have shown that spontaneous magnetization of electrons has more strong effect on galvanomagnetic properties than the phenomena, which are due to spinorbital interaction. The specific behaviors revealed due to spontaneous magnetization must be taken into account in studying the magnetism of semiconductor systems with the transition element impurities what is confirmed by the results of the works [1-3].

\section{Acknowledgements}

The work was performed as a part of the state task FASO Russia (subject "Electron», № 01201463326) with partial support by the RFBR (project №15-02-08909 ), Fundamental Research Program of UB RAS 2015-2017 (project № 15-17-232), by Act 211 Government of the Russian Federation, agreement № 02.A03.21.0006, by the Ministry of Education and Science of the Russian Federation, projects 5719.

\section{References}

1. A.T.Lonchakov, V.I.Okulov, T.E. Govorkova, M.D. Andriychuk, L.D.Paranchich, JETP Letters 96, 405 (2012).

2. A.T. Lonchakov, V.I. Okulov, E.A. Pamyatnykh, T.E. Govorkova, M.A.Andriichuk, L.D. Paranchich, S.B. Bobin and V.V. Deryushkin, Solid State Phenomena 233-234, 456 (2015).

3. Lonchakov, A.T., Okulova, K.A., Pamyatnykh, E.A., Podgornykh, S.M., Andrichuk, M.D., Paranchich, L.D. , Low Temperature Physics 39 , 384 (2013). 